\title{
USO DE BIOCARBÓN EN EL BALANCE DE NITRÓGENO EN SUELOS ALUVIALES DE SAN RAMÓN / CHANCHAMAYO / PERÚ
}

\section{BIOCHAR USE IN THE NITROGEN BALANCE IN ALUVIAL SOILS OF SAN RAMON / CHANCHAMAYO / PERU}

\author{
Mariela Leveau ${ }^{1,2}$, Sebastian Dumler ${ }^{1,3}$, Ruy Anaya De La Rosa ${ }^{4}$, Julio Alegre ${ }^{5}$ y \\ Brenton Ladd ${ }^{1,6}$
}

\begin{abstract}
Resumen
En el Perú se ha incrementado en las últimas décadas el uso intensivo de fertilizantes nitrogenados en los cultivos anuales y perennes. Estos fertilizantes son rápidamente disponibles para las plantas al convertirse en nitratos, incrementando los rendimientos. Sin embargo, los abonos nitrogenados son muy volátiles y se pierden rápidamente también por lixiviación. Este nitrógeno (N) perdido implica costos económicos y también conlleva a problemas ambientales como la eutrofización. El biocarbón ha sido propuesto como una solución a estos problemas, pero se tienen pocas investigaciones sobre el tema en el Perú. El objetivo de este estudio fue evaluar el efecto de la aplicación de biocarbón en el rendimiento de maíz y el balance de nitrógeno en suelos (Entisoles con deposición aluvial) en San Ramón (Perú). Se produjo biocarbón a partir de residuos verdes y estiércol de cerdo. El diseño de la investigación consideró cinco tratamientos: control (T1), 7 t·ha- ${ }^{-1}$ de estiércol de cerdo (T2), $17.5 \mathrm{t} \cdot \mathrm{ha}^{-1}$ de biocarbón (T3), $23 \mathrm{t} \cdot \mathrm{ha}^{-1}$ de biocarbón con ajuste de $\mathrm{pH}$ (T4), y $17.5 \mathrm{t} \cdot \mathrm{ha}^{-1}$ de biocarbón con ajuste de $\mathrm{pH}$ y de relación $\mathrm{C} / \mathrm{N}$ (T5). Los resultados demostraron que los tratamientos T1 y T2 obtuvieron los rendimientos de maíz más bajos, con una producción promedio de 0.41 y $2.45 \mathrm{t} \cdot \mathrm{ha}^{-1}$, respectivamente. El tratamiento $\mathrm{T} 5$ produjo el mayor rendimiento de maíz alcanzando una producción de $5.71 \mathrm{t} \cdot \mathrm{ha}^{-1}$. Sólo se encontró diferencia significativa en el rendimiento de los tratamientos T1 y T5 $(\mathrm{p}=0.011)$. Los porcentajes de eficiencia de uso del nitrógeno (EUN), definida como el porcentaje de $\mathrm{N}$ aplicado que estaba disponible para ser absorbido por el cultivo o retenido en el suelo, fueron mayores para los tratamientos T3, T4 y T5 con 28, 28 y $56 \%$ de eficiencia, respectivamente, en comparación a los tratamientos T1 y T2. Asimismo, los tratamientos con biocarbón T3, T4 y T5 produjeron menores pérdidas de nitrógeno en comparación con los tratamientos T1 y T2. Esta investigación concluyó que las enmiendas con biocarbón tienen efectos positivos en el rendimiento del cultivo del maíz mientras limitan la pérdida de nitrógeno.
\end{abstract}

Palabras clave: enmienda del suelo, estiércol de cerdo, pirólisis, residuos verdes.

\begin{abstract}
In the last decades, the intensive use of nitrogen fertilizers in annual and perennial crops has increased in Peru. These fertilizers are quickly available to plants by converting to nitrates, increasing crop yields. However, nitrogen fertilizers are very volatile and are also lost easily by leaching. This lost nitrogen $(\mathrm{N})$ implies economic costs and leads to environmental problems such as eutrophication. Biochar, a new soil amendment, has been suggested as a solution to these problems; however, there has been little research on the subject in Peru. The objective of this study was to evaluate the effect of biochar application on corn yield and nitrogen balance in soils (Entisols with alluvial deposition) in San Ramon (Peru). Biochar was produced from green waste and pig manure. The research design considered five treatments: Control (T1), $7 \mathrm{t} \cdot \mathrm{ha}^{-1}$ of pig manure (T2), $17.5 \mathrm{t} \cdot \mathrm{ha}^{-1}$ of biochar (T3), $23 \mathrm{t} \cdot \mathrm{ha}^{-1}$ of biochar with $\mathrm{pH}$ adjustment (T4), and $17.5 \mathrm{t} \cdot \mathrm{ha}^{-1}$ of biochar with $\mathrm{pH}$ and $\mathrm{C} / \mathrm{N}$ ratio adjustment (T5). The results showed that treatments $\mathrm{T} 1$ and $\mathrm{T} 2$ obtained the lowest corn yields, with an average production of $0.41 \mathrm{t} \cdot \mathrm{ha}^{-1}$ and $2.45 \mathrm{t} \cdot \mathrm{ha}^{-1}$, respectively. Treatment T5 produced the highest corn yield, reaching a production of $5.71 \mathrm{t} \cdot \mathrm{ha}^{-1}$. A significant difference was only found in the yield of treatments T1 and T5 $(p=0.011)$. Nitrogen use efficiency percentages (NUE), defined as the percentage of applied $\mathrm{N}$ that was available to be absorbed by the crop or retained in the soil, were higher for treatments T3, T4, and T5 with 28, 28, and 56\% efficiency, respectively, in comparison to treatments $\mathrm{T} 1$ and $\mathrm{T} 2$. Likewise, biochar treatments $\mathrm{T} 3$, T4, and T5 produced lower nitrogen losses compared to treatments T1 and T2. This research concluded that biochar-based amendments have positive effects on corn crop yield while limiting nitrogen loss.
\end{abstract}

Key words: soil amendment, pig manure, pyrolysis, green waste. 


\section{Introducción}

En las últimas décadas, los fertilizantes nitrogenados han sido unos componentes críticos para la agricultura; sin embargo, el uso ineficiente de los mismos ha generado pérdidas de alrededor del 50\%, repercutiendo negativamente en el ambiente (Dimkpa et al., 2020; Zhao et al., 2020). En el Perú, el uso de fertilizantes nitrogenados ha tenido un incremento significativo, ya que entre 2001 y 2013 , el volumen de importación se incrementó de 656447 a 905798 toneladas métricas (INEI, 2014).

Los fertilizantes amoniacales, como la urea y los fosfatos monoamónicos y biamónicos, son rápidamente convertidos en nitratos a través del proceso de nitrificación, liberando ácidos $\mathrm{y}$, por lo tanto, incrementando la acidez de la superficie del suelo (Gomero \& Velásquez, 1999; Kanter et al., 2020). La acidez tiene efectos nocivos sobre el crecimiento de las plantas, afecta la disponibilidad de nutrientes y el rendimiento del cultivo (Raza et al., 2020).

Por otro lado, la volatilización de nitrógeno a la atmósfera genera principalmente óxido nítrico, causante de fenómenos como la lluvia ácida, y óxido nitroso, gas de efecto invernadero con potencial de calentamiento 265 veces superior al $\mathrm{CO}_{2}$ (Martínez et al., 2011; IPCC, 2015; Fagodiya et al., 2020).

En el Perú, el uso excesivo de fertilizantes y mal manejo del suelo han causado problemas de salinización, pérdida de la fertilidad y mal drenaje en los suelos (Eguren \& Marapi, 2015). En los últimos 20 años, se ha degradado más del $15 \%$ del territorio peruano que afectó a casi $11 \%$ de la población (INEI, 2013); si esta tendencia se mantiene, el $64 \%$ del territorio peruano podría estar afectado por procesos de esta naturaleza para el 2100 (MINAM, 2014).

Por lo tanto, el desarrollo de tecnologías y estrategias agrícolas que incrementen la productividad de los cultivos sin daños al ambiente es esencial para garantizar la seguridad alimentaria (FAO, 2012). Es así que, el biocarbón podría ofrecer una estrategia para mejorar la calidad y productividad del suelo, la seguridad alimentaria y para mitigar gases de efecto invernadero (Ladd et al., 2017; Werner et al., 2018; Borchard et al., 2019; Banger et al., 2020; Thomas et al., 2020).

El término biocarbón se refiere a la materia orgánica descompuesta térmicamente bajo un suministro limitado de oxígeno (Edmunds, 2012; Ladd et al., 2017). Blackwell et al. (2009) y Sparkes \& Stoutjesdijk (2011) concluyeron que la aplicación de biocarbón mejora el pH del suelo, la capacidad de intercambio catiónico (CIC) y la capacidad de retención de agua. De igual manera, el biocarbón ayuda a retener y mejorar la disponibilidad de los nutrientes como nitrógeno $(\mathrm{N})$ y fósforo $(\mathrm{P})$, y reduce las pérdidas por volatilización y lixiviación (Hagemann et al., 2017; Bolan et al., 2021). Algunos estudios demuestran que la producción y aplicación del biocarbón en el suelo reduce las emisiones de óxidos de nitrógeno (Woolf et al., 2010; Zhang et al., 2010, 2012; Song et al., 2016). La incorporación de biocarbón en el suelo podría regular los procesos del ciclo del nitrógeno, contribuir a mitigar la contaminación de acuíferos por lixiviación de nitratos, reducir las emisiones de óxidos de nitrógeno y aumentar la eficacia del uso de los fertilizantes. Consecuentemente, el rendimiento de los cultivos podría aumentar, pero dichos efectos son variables (Cayuela et al., 2014). Dicha variabilidad podría ser causada por la amplia gama de materias primas utilizadas para fabricar biocarbón (Thomas et al., 2020).

Esta investigación tuvo como objetivo comparar el efecto de diferentes formulaciones de biocarbón sobre el rendimiento de maíz y la pérdida de nitrógeno en el sistema planta-suelo.

\section{Materiales y métodos}

$\underline{\text { Área de estudio }}$

El proyecto se realizó en la estación experimental de la Asociación Peruana para la Promoción del Desarrollo Sostenible (APRODES), en el distrito de San Ramón, Provincia de Chanchamayo, Región Junín, Perú, ubicada a $950 \mathrm{msnm}\left(11^{\circ} 08^{\prime} 22.3^{\prime \prime} \mathrm{S}\right.$ y $75^{\circ} 23^{\prime}$ 44.3 "W). En esta región, los productores de café a pequeña escala (de 1 a 3 ha) cultivan maíz para alimentar a los pollos. El suelo del sitio experimental es un Entisol con deposición aluvial. La caracterización de los suelos del sitio se muestra en la Tabla 1, y fue realizada en el Laboratorio de Análisis de Suelos, Plantas, Aguas y Fertilizantes de la Universidad Nacional Agraria La Molina (UNALM), Lima, Perú.

Tabla 1. Análisis de caracterización del suelo.

\begin{tabular}{llc}
\hline PARÁMETRO & UNIDAD & VALOR \\
\hline $\mathrm{pH}$ & - & 8.35 \\
$\mathrm{Conductividad}$ & $\mathrm{dS} \cdot \mathrm{m}^{-1}$ & 0.18 \\
$\mathrm{CaCO}_{3}$ & $\%$ & 9.1 \\
Materia orgánica & $\%$ & 4.42 \\
$\mathrm{P}$ & $\mathrm{ppm}$ & 5.2 \\
$\mathrm{~K}$ & $\mathrm{ppm}$ & 71 \\
$\mathrm{Arena}$ & $\%$ & 49 \\
$\mathrm{Limo}$ & $\%$ & 38 \\
$\mathrm{CIC}$ & $\mathrm{meq} / 100 \mathrm{~g}$ & 10.08 \\
$\mathrm{Ca}$ & meq/100 g & 8.81 \\
$\mathrm{Mg}^{+2}$ & meq/100 g & 1.02 \\
$\mathrm{Na}^{+}$ & meq/100 g & 0.1 \\
$\mathrm{~K}^{+}$ & $\mathrm{meq} / 100 \mathrm{~g}$ & 0.15 \\
$\mathrm{Al}^{+3}+\mathrm{H}^{+}$ & $\mathrm{meq} / 100 \mathrm{~g}$ & 0 \\
$\mathrm{Fe}$ & $\mathrm{ppm}$ & 40.8 \\
$\mathrm{~N}$ & $\%$ & 0.06 \\
\hline
\end{tabular}

$\underline{\text { Materiales }}$

Se formularon cinco tratamientos: control (T1), estiércol de cerdo (T2), biocarbón (T3), biocarbón con ajuste de $\mathrm{pH}$ (T4), biocarbón con ajuste de $\mathrm{pH}$ y relación de $\mathrm{C} / \mathrm{N}$ (T5). Para el tratamiento T2 se recolectó estiércol de cerdo, el cual se dejó secar a temperatura ambiente $\left(25{ }^{\circ} \mathrm{C}\right)$ por una semana. El 
biocarbón (T3) fue producido a partir de una mezcla proporcional de residuos verdes (materia de poda) y estiércol de cerdo, pirolizado a una temperatura de entre 350 y $550{ }^{\circ} \mathrm{C}$ durante 90 minutos en un horno Kon Tiki de acero con forma de cono profundo (Smebye et al., 2017). El biocarbón (T3) fue alcalino $(\mathrm{pH}=10)$ con una relación $\mathrm{C} / \mathrm{N}$ de 25:1. Para el tratamiento $\mathrm{T} 4$, al biocarbón alcalino (T3) se le adicionó ácido fosfórico para ajustar el pH a 7.5. Para el tratamiento T5 se ajustó la relación $\mathrm{C} / \mathrm{N}$ de $\mathrm{T} 4$ a 10:1 añadiendo 24 gramos de urea por kilogramo de biocarbón. En la Tabla 2 se presentan las características químicas de los tratamientos T2, T3, T4 y T5 de los análisis realizados en el Laboratorio del Departamento de Industrias Primarias de Nueva Gales del Sur en Australia.

Diseño Experimental

Para evaluar el efecto del biocarbón, se sembraron semillas de maíz (Zea mays L.) variedad amarillo duro "Marginal 28 Tropical" (INIA, 2008). El experimento se realizó entre mayo y septiembre del 2016. De acuerdo con las recomendaciones del INIA (2008), se determinó que el área ocupada de cada planta de maíz fuera de $0.4 \mathrm{~m}^{2}\left(0.8 \times 0.5 \mathrm{~m}^{2}\right)$ y la superficie de cada parcela de $4 \mathrm{~m}^{2}$. El diseñó experimental fue de bloques aleatorios. Se hicieron cinco repeticiones por tratamiento. Los tratamientos fueron incorporados al suelo con un azadón y la parcela fue arada con la misma herramienta hasta por $10 \mathrm{~cm}$ y las enmiendas fueron incorporadas en el suelo de forma uniforme. Las parcelas sin enmiendas (control) también fueron aradas. La dosis de aplicación de las enmiendas se formuló de acuerdo con la necesidad nutricional del cultivo de maíz (175 Kg-N·ha-1) recomendada por el Departamento de Industrias Primarias de New South Wales (DPI NSW) en Australia (Beckingham, 2017). Antes de aplicar las enmiendas (T2, T3, T4, T5) se midió el contenido de $\mathrm{N}$ de cada enmienda y se calculó la cantidad necesaria para lograr una tasa de fertilización de $175 \mathrm{Kg}-\mathrm{N} \cdot \mathrm{ha}^{-1}$. Así, las tasas de aplicación de las enmiendas fueron estandarizadas en términos de $\mathrm{N}$ añadido (Tabla 3 ).

Tabla 3. Datos utilizados para calcular y asegurar la estandarización de las tasas de aplicación de nitrógeno a través de los tratamientos.

\begin{tabular}{|c|c|c|c|c|}
\hline 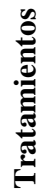 & $\begin{array}{l}\text { Fo } \\
\text { 我 } \\
\mathbf{Z} \\
0 \\
0\end{array}$ & $\mathbf{t} \cdot \mathbf{h a}^{-1 *}$ & 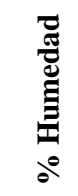 & 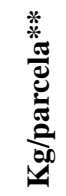 \\
\hline T1 & & & & \\
\hline $\mathrm{T} 2$ & 2.5 & 7 & 26.8 & 3.55 \\
\hline T3 & 1 & 17.5 & 19.2 & 8.34 \\
\hline $\mathrm{T} 4$ & 0.76 & 23.03 & 5.6 & 9.73 \\
\hline T5 & 1 & 17.5 & 11.4 & 7.8 \\
\hline
\end{tabular}

T1 = Control, $\mathrm{T} 2=$ Estiércol de Cerdo, T3 = Biocarbón, T4 = Biocarbón con ajuste de pH y T5 = Biocarbón con ajuste de $\mathrm{pH}$ y relación $\mathrm{C} / \mathrm{N}$. * Aplicación formulada en base al requerimiento nutricional del maíz. ** Dosis de aplicación incluyendo el porcentaje de humedad y el tamaño de la parcela $\left(4 \mathrm{~m}^{2}\right)$.

\section{Parámetros Evaluados y Métodos}

Biomasa Seca: Se extrajeron cinco plantas por parcela y fueron fraccionadas en: hojas, tallos, raíces y mazorcas, y acondicionadas en bolsas de papel. Una vez codificadas, fueron colocadas en la estufa a temperatura constante de $65^{\circ} \mathrm{C}$ por 48 horas hasta obtener un peso constante.

Nitrógeno en el suelo: el contenido de $\mathrm{N}$ total del suelo, antes de la adición de estiércol de cerdo o biocarbón, fue de $0.06 \%$, que representa $900 \mathrm{Kg} \cdot \mathrm{ha}^{-1} \mathrm{de}$

Tabla 2. Características químicas de los tratamientos (T2, T3, T4 y T5).

\begin{tabular}{lccccc}
\hline Parámetro & Unidad & T2 & T3 & T4 & T5 \\
\hline Fósforo & $\%$ & 2 & 1.3 & 1.3 & 1.9 \\
Acido fórmico & $\mathrm{mg} / \mathrm{Kg}$ & 15000 & 12000 & 12000 & 18000 \\
Carbono Orgánico Total & $\%$ & 33 & 16 & 12 & 18 \\
Carbono Total & $\%$ & 33 & 16 & 12 & 18 \\
Nitrógeno Total & $\%$ & 2.5 & 1 & 0.76 & 1 \\
Conductividad Eléctrica & $\mathrm{dS} \cdot \mathrm{m}^{-1}$ & 18 & 6.1 & 5.6 & 8.1 \\
$\mathrm{pH}(\mathrm{CaCl}$ ) & & 7.1 & 7.6 & 7.4 & 7.9 \\
Cationes Intercambiables & & & & & \\
Aluminio & $\mathrm{cmol}(+) / \mathrm{Kg}$ & $<0.1$ & $<0.1$ & $<0.1$ & $<0.1$ \\
Calcio & $\mathrm{cmol}(+) / \mathrm{Kg}$ & 7.2 & 21 & 19 & 6.2 \\
Potasio & $\mathrm{cmol}(+) / \mathrm{Kg}$ & 49 & 18 & 17 & 27 \\
Magnesio & $\mathrm{cmol}(+) / \mathrm{Kg}$ & 21 & 11 & 6.4 & 12 \\
Sodio & $\mathrm{cmol}(+) / \mathrm{Kg}$ & 54 & 16 & 17 & 26 \\
CIC & $\mathrm{cmol}(+) / \mathrm{Kg}$ & 130 & 66 & 59 & 72 \\
Calcio / Magnesio & & 0.35 & 1.9 & 2.9 & 0.51 \\
Calcio Intercambiable & $\%$ & 5.5 & 32 & 31 & 8.6 \\
Potasio Intercambiable & $\%$ & 37 & 27 & 29 & 38 \\
Magnesio Intercambiable & $\%$ & 16 & 17 & 11 & 17 \\
\hline T2= estiércol de cerdo (control de fertilización), T3 $=$ biocarbón, T4 biocarbón con ajuste de pH y T5 \\
= biocarbón con pH y relación C/N ajustado. & & & &
\end{tabular}


$\mathrm{N}$, mientras que el $\mathrm{N}$ agregado por fertilización (Nfer) fue de $175 \mathrm{Kg} \cdot \mathrm{ha}^{-1}$ de $\mathrm{N}$ para todos los tratamientos, excepto para el control. Por lo tanto, para los tratamientos T2, T3, T4 y T5, la cantidad de $\mathrm{N}$ inicial en el suelo fue de $1075 \mathrm{Kg} \cdot \mathrm{ha}^{-1}$ de $\mathrm{N}$.

Para medir la cantidad de $\mathrm{N}$ en el suelo al final del experimento, a los 150 días, se muestreó un $\mathrm{Kg}$ de suelo a la profundidad de $10 \mathrm{~cm}$ para cada parcela. Todas las muestras fueron enviadas un laboratorio de análisis de la UNALM para determinar el $\mathrm{N}$ total por medio del método de Kjeldahl (Bazán, 1996). Las muestras se mantuvieron en la estufa hasta obtener peso constante y se analizaron cinco réplicas por tratamiento.

Nitrógeno en la planta: A los 124 días de siembra, cuando se inició la etapa de floración femenina (estadio V5, según la escala de Ritchie \& Hanway, 1966), se recolectaron muestras de 5 hojas por cada planta para determinar el $\mathrm{N}$ foliar. Dichas hojas fueron ubicadas 3 nodos por debajo del meristemo apical. A los 150 días, con la mazorca desarrollada, se tomaron muestras del resto de las plantas (mazorca, raíz, tallo y hojas), las cuales fueron separadas y secadas en un horno durante tres días a temperatura de $60{ }^{\circ} \mathrm{C}$ (Bazán, 1996); luego, se pesaron las partes del cultivo, para determinar la biomasa total y de las fracciones del cultivo. Una muestra de cada una de las fracciones fue enviada a un laboratorio de la UNALM para determinar la cantidad de $\mathrm{N}$ por el método Kjeldahl (Bazán, 1996). Finalmente, el $\% \mathrm{~N}$ de cada fracción fue multiplicado por la biomasa correspondiente y se determinó el contenido de $\mathrm{N}$ total en el cultivo. Se realizaron 5 réplicas por cada tratamiento.

Balance de Nitrógeno: El balance de masa de $\mathrm{N}$ se realizó considerando las entradas y salidas del sistema suelo-cultivo en cada uno de los tratamientos (Fórmula 1), de acuerdo con Walter et al. (2016).

$$
\mathrm{N}_{\text {entrada }}=\mathrm{N}_{\mathrm{is}}+\mathrm{N}_{\text {fer }}=\mathrm{N}_{\mathrm{ap}}+\mathrm{N}_{\mathrm{fs}}=\mathrm{N}_{\text {salida }}
$$

Fórmula 1. Cálculo del balance de nitrógeno.

Donde:

$\mathrm{N}_{\mathrm{is}}$ : nitrógeno inicial en el suelo.

$\mathrm{N}_{\text {fer }}$ : nitrógeno aportado por fertilización.

$\mathrm{N}_{\mathrm{ap}}$ : nitrógeno absorbido por la planta.

$\mathrm{N}_{\mathrm{fs}}$ : nitrógeno final en el suelo.

$\mathrm{N}_{\text {is }}+\mathrm{N}_{\text {fer }}$ : $\mathrm{N}_{\text {entrada }}$.

$\mathrm{N}_{\mathrm{ap}}+\mathrm{N}_{\mathrm{fs}}: \mathrm{N}_{\text {salida }}$.

Eficiencia del uso de N: La Fórmula 2 representa el método de eficiencia, según Varvel \& Peterson (1990), por medio del cual se calcula la eficiencia del uso de $\mathrm{N}$ y/o la recuperación de fertilizantes en los sistemas de producción de cultivos.

$$
\mathrm{PFR}=\frac{\mathrm{NF}-\mathrm{NC}}{\mathrm{R}}
$$

Fórmula 2. Cálculo de la eficiencia del uso de Nitrógeno.
Donde:

NF : absorción total de $\mathrm{N}$ del cultivo (maíz) de parcelas fertilizadas.

NC : absorción total de $\mathrm{N}$ del cultivo (maíz) de parcelas no fertilizadas.

R : dosis de producto aplicado.

PFR : porcentaje de recuperación. Eficiencia agronómica.

Análisis estadístico

Se utilizó un ANOVA con un $95 \%$ de confianza mediante el software estadístico JMP, v11.0 (SAS institute, [s/f]). El método de comparación para detectar diferencias significativas entre los tratamientos fue Tukey (HSD) con nivel de significación $\leq 0.05$.

\section{Análisis económico}

La rentabilidad de los tratamientos se calculó utilizando los valores medios de rendimiento por hectárea menos sus costos de instalación. Se supuso que el maíz podría venderse a $\mathrm{S} / 1.20 \cdot \mathrm{Kg}^{-1}$ (S/ = símbolo de la moneda peruana, Sol $=$ PEN) usando cifras publicadas por el Ministerio de Agricultura y Riego del Perú (MINAGRI, 2016). Se obtuvo un estimado del rendimiento usando el índice de cosecha de 0.84 para convertir la biomasa de mazorcas en biomasa de granos (Zamudio-González et al., 2016). El costo de la urea $\left(\mathrm{S} / 1.4 \cdot \mathrm{Kg}^{-1}\right)$, el costo del ácido fosfórico $\left(\mathrm{S} / 7.50 \cdot 1^{-1}\right)$ y el costo del biocarbón, se obtuvieron de los cálculos realizados en el marco del proyecto Biochar for Sustainable Soils (B4SS) (Startfish Initiatives, 2018). El tipo de cambio fue 1 USD = 3.4 PEN. En la Tabla 4 se observa el costo de producción de cada tratamiento.

\section{Resultados}

Las enmiendas evaluadas han tenido efectos significativos en el rendimiento de maíz (Tabla 5, Figura 1). Con la adición de estiércol de cerdo se aumentó el rendimiento de maíz significativamente por un factor de 5. Los resultados, consistentes con la hipótesis planteada, mostraron efectos muy variados y no fueron iguales para los diferentes tipos de tratamientos con biocarbón. El biocarbón más sofisticado (T5) fue el más efectivo, aumentando significativamente los rendimientos y crecimiento de las plantas de maíz (Tabla 6, Figuras 1 y 2a). Los contenidos de $\mathrm{N}$ en las plantas y suelos indican que el biocarbón mejoró la eficiencia en el uso de N (Tabla 5, Figura 2b-e). Es importante señalar que la formulación de biocarbón que ha resultado en una mejor eficiencia de uso de nitrógeno (EUN) (Tablas 7 y 8) pudo ser debido a su efecto positivo en el crecimiento del cultivo, habiéndose aprovechado con mayor eficiencia el nutriente añadido con las enmiendas y reduciéndose las pérdidas. En términos de rentabilidad, la única enmienda que sale rentable en el sistema de cultivo de maíz es el que contiene estiércol de cerdo, ya que triplicó la rentabilidad del cultivo (Tabla 9). Ninguna 
de las otras tres formulaciones de biocarbón fueron rentables para el cultivo de maíz (Tabla 9).

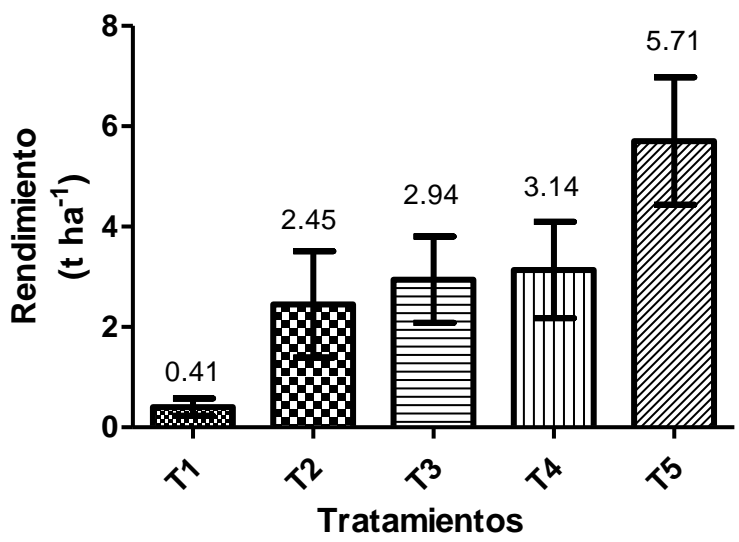

$\mathrm{T} 1$ = Control, $\mathrm{T} 2$ = Estiércol de Cerdo, T3 = Biocarbón, $\mathrm{T} 4=$ Biocarbón con ajuste de $\mathrm{pH}$ y T5 = Biocarbón con ajuste de $\mathrm{pH}$ y relación $\mathrm{C} / \mathrm{N}$.

Figura 1. Rendimiento de maíz en cada uno de los tratamientos expresados en toneladas por hectárea (Promedio \pm Desviación Estándar).

\section{Discusión}

La acumulación de biomasa y $\mathrm{N}$ en los tratamientos varió manteniendo la tendencia $\mathrm{T} 1<\mathrm{T} 2<\mathrm{T} 3<\mathrm{T} 4<$ T5. Sabiendo que la acumulación de $\mathrm{N}$ en la biomasa aérea de las plantas de maíz varía entre 200 y 300 $\mathrm{Kg} \cdot \mathrm{ha}^{-1}$ (Rodríguez et al., 2014), la baja absorción de $\mathrm{N}$ observada pudo deberse a múltiples factores como, por ejemplo, la falta de agua debido a que al maíz necesita suficiente cantidad de agua disponible para que se pueda asimilar nitrógeno en forma de nitrato (Rodríguez et al., 2014). Un estudio demostró que el biocarbón aumenta la humedad del suelo y así favorece la absorción de N (Cayuela et al., 2014) y es importante evaluarlo en investigaciones futuras. Por otro lado, los estudios llevados a cabo por la FAO (2002) mostraron que sin la aplicación de fósforo y potasio la eficiencia del uso de $\mathrm{N}$ en el maíz disminuye mientras que, cuando se aplican todos los macronutrientes la eficiencia del uso de $\mathrm{N}$ aumenta de manera sostenida, indicando la interacción entre los nutrientes. El mayor crecimiento y EUN de maíz en T5 podría estar vinculado con una nutrición más óptima de fósforo o potasio, pero estos elementos no fueron evaluados en este estudio y es otro tema importante para investigaciones futuras.

Los principales mecanismos de pérdida de $\mathrm{N}$ son: lixiviación, especialmente $\mathrm{N}^{-} \mathrm{NO}_{3}^{-}$, volatilización de $\mathrm{NH}_{4}{ }^{+}$y desnitrificación con la producción de gases $\left(\mathrm{N}_{2} \mathrm{O}^{-}\right.$y $\mathrm{N}_{2}$ ) hacia la atmósfera (Borchard et al., 2019). Subbarao et al. (2006) indicaron que la fuente más importante de pérdida de $\mathrm{N}$ en el suelo en forma gaseosa es la desnitrificación, donde los nitratos se reducen a óxido nitroso $\left(\mathrm{N}_{2} \mathrm{O}^{-}\right)$con la consecuente liberación del $\mathrm{N}$ en su forma elemental gaseosa $\left(\mathrm{N}_{2}\right)$.
Este proceso es importante durante períodos con altas precipitaciones en suelos con altas temperaturas y con un alto contenido de materia orgánica. Estas condiciones meteorológicas son características del área de estudio, pero el método de balance de masas utilizado en este estudio no permitió sacar conclusiones concretas con relación a los mecanismos de pérdidas de nitrógeno, desnitrificación, volatilización y lixiviación. Lo que se podría inferir es que la alta EUN en el T5 (la formulación de biocarbón más sofisticada) fue debida a que esta enmienda ha promovido el mayor crecimiento de las plantas. Esto permitió una rápida absorción del $\mathrm{N}$ aplicado y que ya no estuvo en el suelo porque, en caso contrario, los procesos bióticos o abióticos podrían haber incentivado la pérdida de este nutriente bastante móvil en el suelo.

A pesar de que las parcelas con biocarbón (T3, T4 y T5) excedieron la producción de T2 (estiércol de cerdo) y T1 (control), el uso de biocarbón no salió rentable en el análisis económico. Esto debido al costo de producción del biocarbón. Bach et al. (2016) estudiaron el uso potencial del biocarbón en la agricultura desde la perspectiva de la economía agrícola y concluyeron que el agricultor no recibirá ningún beneficio económico si solo consideramos el valor agronómico del biocarbón en el suelo. Por esta razón, se recomienda el uso de incentivos financieros como los bonos de carbono para subsidiar los costos vinculados con el uso del biocarbón. También, podríamos analizar los costos de producción. Quizás aumentando la producción a mayor escala podríamos reducir el costo del biocarbón, haciéndolo más viable para el agricultor. Otra posibilidad es usar biocarbón como un coproducto para fertilizantes convencionales; esto es rentable (Ladd et al., 2017). Por otro lado, los agricultores podrían producir su propio biocarbón (sin costo) utilizando biomasa residual que obtienen de la limpieza de sus huertos. Dichas estrategias deberían ser el enfoque de futuras investigaciones.

\section{Conclusiones}

Las enmiendas en base a biocarbón tienen un efecto positivo en el rendimiento del cultivo del maíz, y la pérdida de nitrógeno resulta ser menor cuando se aplica biocarbón al suelo. A fin de poder obtener más información respecto al efecto del biocarbón en los nichos para la flora microbiana, se recomienda evaluar la biótica del biocarbón. Es preciso resaltar que los tratamientos en base a biocarbón no resultaron ser rentables debido al elevado costo de su producción. Sabiendo que el biocarbón mejora la producción del maíz, se tienen que buscar mejores estrategias para lograr el mayor potencial de mitigación al cambio climático de esta enmienda y será importante investigar y desarrollar estrategias que hagan económicamente viable el uso del biocarbón para el agricultor de pequeña escala. 


\section{Agradecimientos}

Este estudio fue parte del proyecto Biochar for Sustainable Soils (B4SS) financiado por el Global Environment Facility

(https://biochar.international/the-biochar-forsustainable-soils-b4ss-project/peru/).

\section{Literatura citada}

Bach M., Wilske B. \& Breuer L. 2016. Current economic obstacles to biochar use in agriculture and climate change mitigation. Carbon Management, 7(3-4): 183-190. https://doi.org/10.1080/17583004.2016.1213608.

Banger K., Wagner-Riddle C., Grant B.B., Smith W.N., Drury C. \& Yang J. 2020. Modifying fertilizer rate and application method reduces environmental nitrogen losses and increases corn yield in Ontario. Science of The Total Environment, 722: 137851. https://doi.org/10.1016/j.scitotenv.2020.137851.

Bazán R. 1996. Manual para el análisis químico de suelos, aguas, plantas. UNALM. Fundación Perú. Lima. 1-7.

Blackwell P., Riethmuller G. \& Collins M. 2009. Biochar application to soil. En: Lehmann J. \& Joseph S. Biochar for Environmental Management: Science and Technology. 207-226. Earthscan Publishing for a sustainable future. UK and USA. http://www.css.cornell.edu/faculty/lehmann/publ/First\% 20proof\%2013-01-09.pdf.

Bolan N., Hoang S.A., Beiyuan J., Gupta S., Hou D., Karakoti A., Joseph S., Jung S., Kim K.-H., Kirkham M.B., Kua H.W., Kumar M., Kwon E.E., Ok Y.S., Perera V., Rinklebe J., Shaheen S.M., Sarkar B., Sarmah A.K., Singh B.P., Singh G., Tsang D.C.W., Vikrant K., Vithanage M., Vinu A., Wang H., Wijesekara H., Yan Y., Younis S.A. \& Van Zwieten L. 2021. Multifunctional applications of biochar beyond carbon storage. International Materials Reviews, 1-51. https://doi.org/10.1080/09506608.2021.1922047.

Borchard N., Schirrmann M., Cayuela M.L., Kammann C., Wrage-Mönnig N., Estavillo J.M., Fuertes-Mendizábal T., Ippolito J. \& Novak J. 2019. Biochar, soil and landuse interactions that reduce nitrate leaching and $\mathrm{N} 2 \mathrm{O}$ emissions: a meta-analysis. Science of the Total Environment, 651(Part 2354-2364. https://doi.org/10.1016/j.scitotenv.2018.10.060.

Beckingham C. 2017. Sweet corn growing. DPI (Department of Primary Industries) / NSW / Australia. https://www.dpi.nsw.gov.au/agriculture/horticulture/veg etables/commodity-growing-guides/sweet-corn.

Cayuela M.L., Van Zwieten L., Singh B.P., Jeffery S., Roig A. \& Sánchez-Monedero M.A. 2014. Biochar's role in mitigating soil nitrous oxide emissions: A review and meta-analysis. Agriculture, Ecosystems \& Environment, 191: 5-16. https://doi.org/10.1016/j.agee.2013.10.009.

Eguren F. \& Marapi R. 2015. Los Suelos en el Perú. Recurso fundamental para crear y sostener vida. La Revista Agraria, (170): 6-10. CEPES.

https://vlex.com.pe/vid/suelos-peru-recursofundamental-732777989.

Dimkpa C.O., Fugice J., Singh U. \& Lewis T.D. 2020 Development of fertilizers for enhanced nitrogen use efficiency - Trends and perspectives. Science of The Total Environment, 731: 139113 https://doi.org/10.1016/j.scitotenv.2020.139113.
Edmunds C.W. 2012. The Effects of Biochar Amendment to Soil on Bioenergy Crop Yield and Biomass Composition. A Thesis Presented for the Master of Science Degree. University of Tenesse. Knoxville. https://trace.tennessee.edu/cgi/viewcontent.cgi?article=2 $316 \&$ context=utk_gradthes.

Fagodiya R.K., Pathak H., Bhatia A., Jain N., Kumar A. \& Malyan S.K. 2020. Global warming impacts of nitrogen use in agriculture: an assessment for India since 1960. Carbon Management, 11(3): 291-301. https://doi.org/10.1080/17583004.2020.1752061.

FAO. 2002. Los fertilizantes y su uso. Cuarta Edición. FAO e IFA (Asociación Internacional de la industria de los fertilizantes). Italia.

https://www.fao.org/documents/card/es/c/b0f8bfc54c95-54b0-80cd-96b810006037/.

FAO. 2012. Hacia el futuro que queremos. Erradicación del hambre y transición a sistemas agrícolas y alimentarios sostenibles. FAO. Italia.

https://cenida.una.edu.ni/relectronicos/REE10O68hf.pdf.

Gomero L. \& Velásquez H. 1999. Manejo Ecológico de Suelo. Conceptos, Experiencias y Técnicas. RAAA (Red de Acción en Alternativas al uso de Agroquímicos). Lima/Perú.

https://raaa.org.pe/storage/uploads/posts/post_20200318 184410.pdf.

Hagemann N., Joseph S., Schmidt H.P., Kammann C.I., Harter J., Borch T., Young R.B., Varga K., Taherymoosavi S., Elliott K.W., McKenna A., Albu M., Mayrhofer C., Obst M., Conte P., Dieguez-Alonso A., Orsetti S., Subdiaga E., Behrens S. \& Kappler A. 2017. Organic coating on biochar explains its nutrient retention and stimulation of soil fertility. Nature communications, 8(1): 1089. https://doi.org/10.1038/s41467-017-01123-0.

INEI. 2013. Territorio y Suelos. En: INEI Perú: Anuario de Estadísticas Ambientales 2013. 27-58. INEI (Instituto nacional de Estadística e Informática). Perú. https://www.inei.gob.pe/media/MenuRecursivo/publicac iones_digitales/Est/Lib1140/Libro.pdf.

INEI. 2014. Capítulo 12: Agrario. En: INEI Compendio Estadístico Perú 2014. 941-1021. INEI (Instituto nacional de Estadística e Informática). Perú. https://www.inei.gob.pe/media/MenuRecursivo/publicac iones_digitales/Est/Lib1173/cap12/ind12.htm.

INIA. 2008. Maíz amarillo duro marginal 28 tropical. INIA (Instituto Nacional de Innovación Agraria). Perú. http://repositorio.inia.gob.pe/handle/20.500.12955/626.

IPCC. 2015. Climate Change 2014: Synthesis Report. IPCC (Intergovernmental Panel on Climate Change). Geneva / Switzerland. https://archive.ipcc.ch/pdf/assessmentreport/ar5/syr/SYR_AR5_FINAL_full_wcover.pdf.

Kanter D.R., Bartolini F., Kugelberg S., Leip A., Oenema O. \& Uwizeye A. 2020. Nitrogen pollution policy beyond the farm. Nature Food, 1(1): 27-32. https://doi.org/10.1038/s43016-019-0001-5.

Ladd B., Dumler S., Loret de Mola E., Anaya de la Rosa R. \& Borchard N. 2017. Incremento de rentabilidad en producción del maíz en Perú: N-fertilizantes y biochar. The Biologist (Lima), 15(2): 351-358. https://doi.org/10.24039/rtb2017152194. 
Martínez F., Ojeda D., Hernández A., Martínez J. \& Quezada G. 2011. Exceso de Nitratos: Un problema actual en la agricultura. Synthesis, 57: 11-16. Facultad de Ciencias Agrotecnólogicas / Universidad Autónoma de Chihuahua.

MINAGRI. 2016. Boletín de maíz amarillo duro 2016, 19 de agosto 2016. MINAGRI (Ministerio de Agricultura y Riego). Perú. https://www.midagri.gob.pe/portal/boletinde-maiz-amarillo-duro/maiz-2016?download=9668:19de-agosto-2016\&start $=80$.

MINAM. 2014. Informe Nacional del Estado del Ambiente 2012-2013. MINAM (Ministerio del Ambiente). Perú. (Página 21).

https://sinia.minam.gob.pe/documentos/informenacional-estado-ambiente-2012-2013.

Raza S., Miao N., Wang P., Ju X., Chen Z., Zhou J. \& Kuzyakov Y. 2020. Dramatic loss of inorganic carbon by nitrogen-induced soil acidification in Chinese croplands. Global change biology, 26(6): 3738-3751. https://doi.org/10.1111/gcb.15101.

Ritchie S. \& Hanway J. 1966. How a corn plant develops. lowa Sate University of Science and Technology \& United States Department of Agriculture. lowa. Special Report $\mathrm{N}^{\circ} 48$. https://core.ac.uk/download/pdf/83024409.pdf.

Rodríguez L., Sombrero A. \& Cedrum M. 2014. Efecto de la fertilización nitrogenada en el cultivo de maíz. Comparación de la siembra directa y el laboreo convencional: determinación de las pérdidas de fertilizantes nitrogenados mediante lixiviación de nitratos. Tierras, 223: 44-51. https://chil.me//downloaddoc/132517.

SAS Institute. [s/f]. JMP v.11.0. In: Statistical Discovery From SAS. SAS Institute Inc. www.jmp.com/es_pe/home.html.

Smebye A.B., Sparrevik M., Schmidt H.P. \& Cornelissen G. 2017. Life-cycle assessment of biochar production systems in tropical rural areas: Comparing flame curtain kilns to other production methods. Biomass and Bioenergy, 101: 35-43. https://doi.org/10.1016/j.biombioe.2017.04.001.

Song X., Pan G., Zhang C.H., Zhang L. \& Wang H. 2016. Effects of Biochar application on fluxes of three biogenic greenhouse gases: a meta-analysis. Ecosystem Health and Sustainability, 2(2): e01202. https://doi.org/10.1002/ehs2.1202.

Sparkes J. \& Stoutjesdijk P. 2011. Biochar: Implications for agricultural productivity. Technical report 11.06. December 2011. Australian Government $\backslash$ Department of Agriculture, Fisheries and Forestry $\backslash$ Australian Bureau of Agricultural and Resource Economics and Sciences (ABARES). Commonwealth of Australia. https://www.melonsaustralia.org.au/wpcontent/uploads/2018/12/Biochar-implications-foragricultural-productivity.pdf.

Startfish Initiatives. 2018. BIOCHAR FOR SUSTAINABLE SOILS (B4SS) PURPOSE. Starfish Initiavites. https://www.biochar.international/.
Subbarao G.V., Ito O., Sahrawat K.L., Berry W.L., Nakahara K., Ishikawa T., Watanabe T., Suenaga K., Rondon M. \& Rao I.M. 2006. Scope and Strategies for Regulation of Nitrification in Agricultural Systems-Challenges and Opportunities. Critical Reviews in Plant Sciences, 25(4): 303-335. https://doi.org/10.1080/07352680600794232.

Thomas E., Borchard N., Sarmiento C., Atkinson R. \& Ladd B. 2020. Key factors determining biochar sorption capacity for metal contaminants: a literature synthesis. Biochar, 2: 151-163. https://doi.org/10.1007/s42773020-00053-3.

Varvel G.E. \& Peterson T.A. 1990. Nitrogen fertilizer recovery by corn in monoculture and rotation systems. Agronomy Journal, 82(5): 935-938. https://doi.org/10.2134/agronj1990.00021962008200050 019x.

Walter S., Giletto C. \& Echeverría H. 2016. Balance de Masa de Nitrógeno en el cultivo de papa en Villa Dolores, Córdoba. Ciencia del Suelo, 34(1): 93-104. http://www.suelos.org.ar/publicaciones/volumen341201 6/93-

$104 \% 20 \mathrm{p} \% \mathrm{C} 3 \% \mathrm{~A} 1 \mathrm{gs} \% 20 \mathrm{CS} \% 20380 \% 20$ Walter\%20ju nio\%2025.pdf.

Werner C., Schmidt H.P., Gerten D., Lucht W. \& Kammann C. 2018. Biogeochemical potential of biomass pyrolysis systems for limiting global warming to $1.5{ }^{\circ} \mathrm{C}$. Environmental Research Letters, 13(4): 044036. https://iopscience.iop.org/article/10.1088/17489326/aabb0e.

Woolf D., Amonette J., Street-Perrott A., Lehmann J. \& Joseph S. 2010. Sustainable Biochar to mitigate global climate change. Nature Communications, 1: 56. https://doi.org/10.1038/ncomms1053.

Zamudio-González B., Tadeo-Robledo M., EspinosaCalderón A., Martínez-Rodríguez N. \& TurrentFernández A. 2016. Índice de cosecha con macronutrimentos en grano de maíz. Revista mexicana de $\begin{array}{llll}\text { ciencias agrícolas, } & \text { 7(5): } & \text { 1077-1089. }\end{array}$ https://doi.org/10.29312/remexca.v7i5.233.

Zhang A., Cui L., Pan G., Li L., Hussain Q., Zhang X., Zheng J. \& Crowley D. 2010. Effect of Biochar amendment on yield and methane and nitrous oxide emissions from a rice paddy from Tai Lake Plain, China. Agriculture, Ecosystems \& Environment, 139(4): 469-475. https://doi.org/10.1016/j.agee.2010.09.003.

Zhang A., Liu Y., Pan G., Hussain Q., Li L., Zheng W. \& Zhang X. 2012. Effect of Biochar amendment on maize yield and greenhouse gas emissions from a soil organic carbon poor calcareous loamy soil from Central China Plain. Plant Soil, 351(1-2): 263-375. https://doi.org/10.1007/s11104-011-0957-x.

Zhao S., Schmidt S., Qin W., Li J., Li G. \& Zhang W. 2020. Towards the circular nitrogen economy - A global metaanalysis of composting technologies reveals much potential for mitigating nitrogen losses. Science of The Total Environment, 704: 135401. https://doi.org/10.1016/j.scitotenv.2019.135401. 
a)

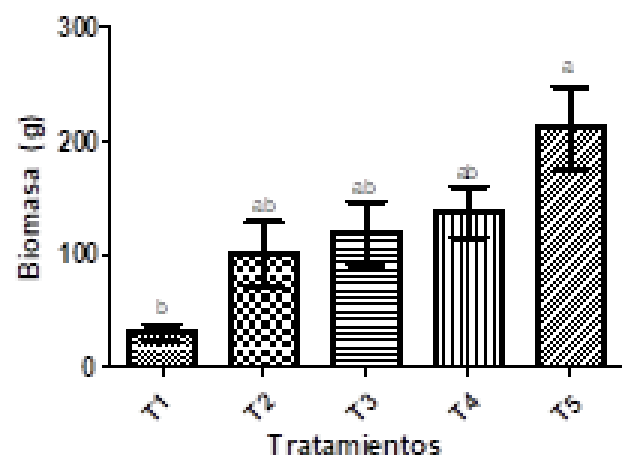

c)

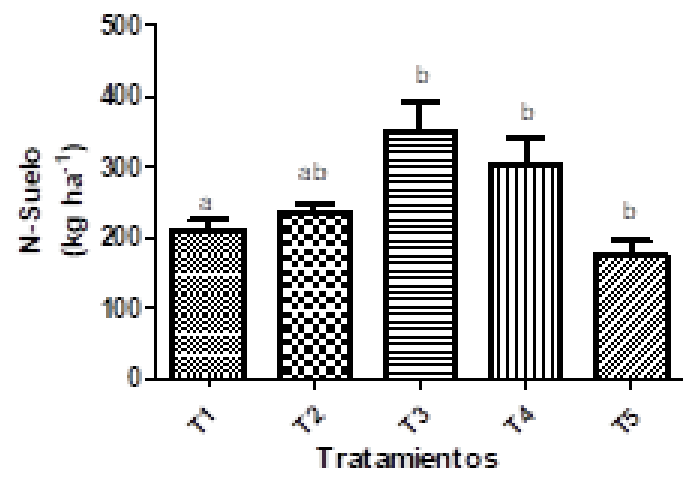

e)

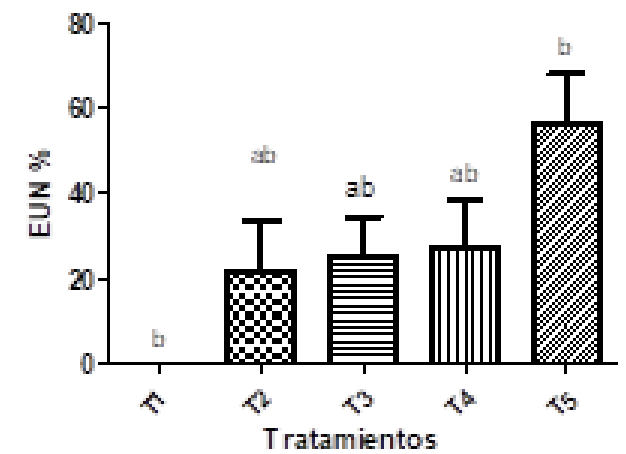

b)

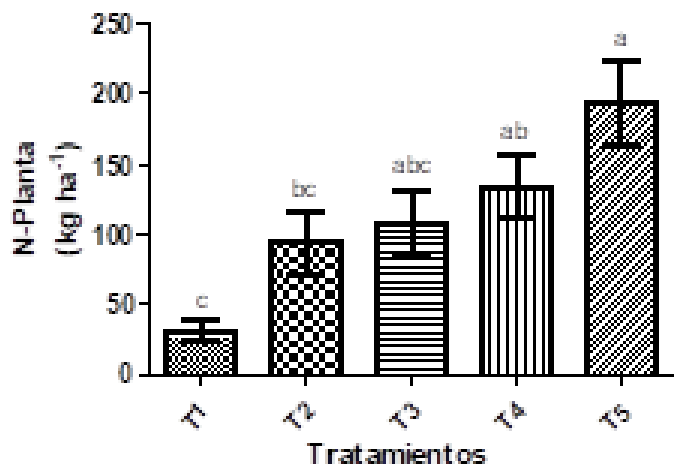

d)

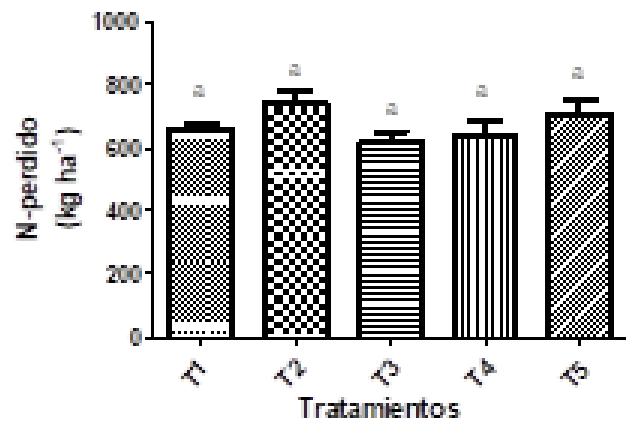

$\mathrm{T} 1$ = Control, $\mathrm{T} 2$ = Estiércol de Cerdo, T3 = Biocarbón, T4 = Biocarbón con ajuste de pH y T5 = Biocarbón con ajuste de pH y relación C/N . Las columnas representan los Promedios \pm Desviación Estándar. Los valores con la misma letra entre distintas columnas no son significativamente diferentes $(\mathrm{P}<0.05)$.

Figura 2. a) Biomasa. b) Nitrógeno en la planta. c) Nitrógeno en el Suelo al final del experimento d) Nitrógeno perdido. e) Eficiencia del uso del Nitrógeno. 
Tabla 4. Costos de los materiales utilizados como enmiendas en el experimento.

\begin{tabular}{|c|c|c|c|c|c|}
\hline Insumo & Unidades & $\begin{array}{r}\text { Precio } \\
\text { Unitario } \\
(\mathrm{S} /)\end{array}$ & $\begin{array}{r}\text { Insumo } \\
\text { por } \\
\text { tonelada de } \\
\text { tratamiento }\end{array}$ & $\begin{array}{r}\text { Costo por } \\
\text { tonelada de } \\
\text { enmienda } \\
(\mathrm{S} /) \\
\end{array}$ & $\begin{array}{r}\text { Costo para } \\
\text { lograr una tasa } \\
\text { de fertilización } \\
176 \mathrm{Kg} \cdot \mathrm{ha}^{-1} \text { de } \mathrm{N}\end{array}$ \\
\hline \multicolumn{6}{|l|}{ TRATAMIENTO 1} \\
\hline Control & & 0 & 0 & 0 & \\
\hline Total & & & & 0 & 0 \\
\hline \multicolumn{6}{|c|}{ TRATAMIENTO 2 (Estiércol de cerdo) } \\
\hline Estiércol de Cerdo & $\mathrm{Kg}$ & 0.2 & 1000 & 200 & \\
\hline Total & & & & 200 & 1400 \\
\hline \multicolumn{6}{|c|}{ TRATAMIENTO 3 (Biocarbón) } \\
\hline Estiércol de Cerdo & $\mathrm{Kg}$ & 0.3 & 600 & 180 & \\
\hline Residuos Verdes & $\mathrm{Kg}$ & 0.4 & 600 & 240 & \\
\hline Mano de Obra & día & 60 & 5 & 300 & \\
\hline Total & & & & 720 & 12600 \\
\hline \multicolumn{6}{|c|}{ TRATAMIENTO 4 (Biocarbón con pH ajustado) } \\
\hline Estiércol de Cerdo & $\mathrm{Kg}$ & 0.3 & 600 & 180 & \\
\hline Residuos Verdes & $\mathrm{Kg}$ & 0.4 & 600 & 240 & \\
\hline Ácido fosfórico & 1 & 7.5 & 12 & 90 & \\
\hline Mano de Obra & día & 60 & 5 & 300 & \\
\hline Total & & & & 810 & 18654 \\
\hline \multicolumn{6}{|c|}{ TRATAMIENTO 5 (Biocarbón con pH y relación C/N ajustado) } \\
\hline Estiércol de Cerdo & $\mathrm{Kg}$ & 0.3 & 600 & 180 & \\
\hline Residuos Verdes & $\mathrm{Kg}$ & 0.4 & 600 & 240 & \\
\hline Ácido fosfórico & 1 & 7.5 & 12 & 90 & \\
\hline Úrea & $\mathrm{Kg}$ & 3.4 & 24 & 81.6 & \\
\hline Mano de Obra & día & 60 & 5 & 300 & \\
\hline Total & & & & 981.6 & 17178 \\
\hline
\end{tabular}

Tabla 5. Resumen del análisis estadístico.

\begin{tabular}{|c|c|c|c|c|}
\hline Fuente & GL* & SC* & F Ratio & Prob. $>$ F \\
\hline \multicolumn{5}{|c|}{ a) Biomasa Total } \\
\hline Tratamientos & 4 & 83097.6 & 6.39 & 0.0022 \\
\hline Error & 18 & 58547.5 & & \\
\hline C. Total & 22 & 141645.1 & & \\
\hline \multicolumn{5}{|c|}{ b) Nitrógeno contenido en plantas } \\
\hline Tratamientos & 4 & 69733.6 & 7.56 & 0.0009 \\
\hline Error & 18 & 41527.6 & & \\
\hline C. Total & 22 & 111261.2 & & \\
\hline \multicolumn{5}{|c|}{ c) Nitrógeno contenido en el suelo } \\
\hline Tratamientos & 4 & 102726 & 6 & 0.0024 \\
\hline Error & 20 & 85590 & & \\
\hline C. Total & 24 & 188316 & & \\
\hline \multicolumn{5}{|c|}{ d) Nitrógeno perdido } \\
\hline Tratamientos & 4 & 48995 & 1.82 & 0.1681 \\
\hline Error & 18 & 120836.5 & & \\
\hline C. Total & 22 & 169831.5 & & \\
\hline \multicolumn{5}{|c|}{ e) Eficiencia de uso de nitrógeno (EUN) } \\
\hline Tratamiento & 4 & 7957 & 4.46 & 0.0111 \\
\hline Error & 18 & 8022.5 & & \\
\hline C. Total & 22 & 15979.5 & & \\
\hline
\end{tabular}


Tabla 6. Biomasa seca total y por fracciones, por tratamiento. Valores de biomasa total promedio \pm el error estándar.

\begin{tabular}{cccccc}
\hline \multirow{2}{*}{ Tratamientos } & \multicolumn{3}{c}{ Fracciones de Biomasa Seca $(\mathrm{g})$} & \multirow{2}{*}{ Biomasa Seca Total $(\mathrm{g})$} \\
\cline { 2 - 5 } & Mazorcas & \multicolumn{1}{c}{ Hojas } & \multicolumn{1}{c}{ Tallo } & \multicolumn{1}{c}{ Raíces } & \\
\hline T1 & 8.09 & 11.06 & 7.88 & 3.78 & $30.81 \pm 6.67$ \\
T2 & 49 & 18.86 & 20.54 & 8.74 & $99.95 \pm 28.2$ \\
T3 & 58.86 & 23.86 & 22.95 & 12.31 & $117.99 \pm 27.77$ \\
T4 & 62.75 & 25.12 & 24.42 & 12.32 & $136.94 \pm 22.67$ \\
T5 & 114.12 & 34.06 & 40.67 & 21.22 & $210.06 \pm 36.41$ \\
\hline
\end{tabular}

T1 = Control, T2 = Estiércol de Cerdo, T3 = Biocarbón, T4 = Biocarbón con ajuste de pH y T5 = Biocarbón con ajuste de $\mathrm{pH}$ y relación $\mathrm{C} / \mathrm{N}$.

Tabla 7. Nitrógeno perdido promedio por tratamiento calculado a partir del balance de nitrógeno en el sistema suelo - planta. Nitrógeno de entrada menos el nitrógeno absorbido por la planta (Nap), el nitrógeno final del suelo (Nfs) y el nitrógeno perdido.

\begin{tabular}{|c|c|c|c|c|c|}
\hline Tratamientos & $\begin{array}{c}\text { N-entrada } \\
\left(\text { Kg }^{\prime} \mathbf{h a}^{-1}\right)\end{array}$ & $\begin{array}{c}\text { Nap } \\
\left(\mathrm{Kg}^{\prime} \mathbf{h a}^{-1}\right)\end{array}$ & $\begin{array}{c}\text { Nfs } \\
\left(\mathrm{Kg}^{\prime} \cdot \mathrm{ha}^{-1}\right)\end{array}$ & $\begin{array}{l}\text { N-perdido } \\
\left(\text { Kg }^{\prime} \text { ha }^{-1}\right)\end{array}$ & $\begin{array}{c}\text { N-perdido } \\
(\%)\end{array}$ \\
\hline $\mathrm{T} 1$ & 900 & 31.36 & 210 & 658.64 & 73.19 \\
\hline $\mathrm{T} 2$ & 1075 & 93.76 & 234 & 744.99 & 69.3 \\
\hline $\mathrm{T} 3$ & 1075 & 108.52 & 351 & 615.48 & 57.25 \\
\hline $\mathrm{T} 4$ & 1075 & 134.02 & 303 & 637.23 & 59.28 \\
\hline T5 & 1075 & 194.33 & 174 & 706.67 & 65.74 \\
\hline
\end{tabular}

T1 = Control, T2 = Estiércol de Cerdo, T3 = Biocarbón, T4 = Biocarbón con ajuste de pH y T5 = Biocarbón con ajuste de $\mathrm{pH}$ y relación $\mathrm{C} / \mathrm{N}$.

Tabla 8. Cálculo de la eficiencia de uso de $\mathrm{N}$ utilizando el método de diferencia.

\begin{tabular}{|c|c|c|c|c|c|}
\hline Tratamiento & $\begin{array}{c}\begin{array}{c}\text { Aplicación N } \\
\text { Kg} \cdot \mathbf{h a}^{-1}\end{array} \\
\end{array}$ & 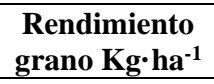 & N-Grano \% & $\begin{array}{c}\text { N absorbido } \\
\text { Kg}^{\prime} \text { ha }^{-1}\end{array}$ & $(\mathbf{E U N}) \%$ \\
\hline T1 & 0 & 404.6 & 2.07 & 8.78 & 0 \\
\hline $\mathrm{T} 2$ & 175 & 2449.75 & 2.04 & 47.81 & 21.29 \\
\hline $\mathrm{T} 3$ & 175 & 2942.8 & 1.93 & 52.67 & 25.08 \\
\hline T4 & 175 & 3137.67 & 1.81 & 57.5 & 26.83 \\
\hline T5 & 175 & 5705.63 & 1.86 & 106.67 & 55.94 \\
\hline
\end{tabular}

T1 = Control, T2 = Estiércol de Cerdo, T3 = Biocarbón, T4 = Biocarbón con ajuste de pH y T5 = Biocarbón con ajuste de $\mathrm{pH}$ y relación $\mathrm{C} / \mathrm{N}, \mathrm{EUN}=$ Eficiencia de uso de nitrógeno.

Tabla 9. Resultados del análisis económico.

\begin{tabular}{llccccc}
\hline Primer ciclo de cultivo & Unidad & T1 & T2 & T3 & T4 & T5 \\
\hline Dosis de aplicación por tratamiento & $\mathrm{t} \cdot \mathrm{ha}^{1}$ & 0 & 7 & 17.5 & 23.02 & 17.5 \\
Costo de aplicación por tonelada & $\mathrm{S} / \cdot \mathrm{t}^{-1}$ & 0 & 200 & 720 & 810 & 1000 \\
Costo de aplicación por hectárea & $\mathrm{S} / \mathrm{ha}^{-1}$ & 0 & 1400 & 12600 & 18646.2 & 17500 \\
Rendimiento del maíz & $\mathrm{t} \cdot \mathrm{ha}^{-1}$ & 0.41 & 2.45 & 2.94 & 3.14 & 5.71 \\
Precio de venta del maíz & $\mathrm{S} / \mathrm{t}^{-1}$ & 1250 & 1250 & 1250 & 1250 & 1250 \\
Ganancias por hectárea & $\mathrm{S} / \cdot \mathrm{ha}^{-1}$ & 512.5 & 3062.5 & 3675 & 3925 & 7137.5 \\
Beneficio neto por hectárea & $\mathrm{S} / \cdot \mathrm{ha}^{-1}$ & 512.5 & 1662.5 & -8925 & -14721.2 & -10362.5 \\
\hline
\end{tabular}

T1 = Control, T2 = Estiércol de Cerdo, T3 = Biocarbón, T4 = Biocarbón con ajuste de pH y T5 = Biocarbón con ajuste de pH y relación $\mathrm{C} / \mathrm{N}$.

\footnotetext{
${ }^{1}$ Universidad Científica del Sur \Facultad de Ciencias Ambientales. Panamericana Sur km 19. Lima / Perú.

2 mariela_192@hotmail.com.

3 jsdg43@ hotmail.com.

${ }^{4}$ Starfish Initiatives, Armidale, NSW 2350, Australia. ruy@biochar.international.

${ }^{5}$ Departamento de Suelos / Universidad Nacional Agraria La Molina. Lima / Perú. jalegre@lamolina.edu.pe.

${ }^{6}$ Autor de contacto: bladd@ucientifica.edu.pe.
} 